\title{
The Feasibility of an Efficient Drug Design Method with High- Performance Computers
}

\author{
Takefumi Yamashita, ${ }^{*, a}$ Akihiko Ueda, ${ }^{b}$ Takashi Mitsui, ${ }^{a, b}$ Atsushi Tomonaga, ${ }^{b}$ \\ Shunji Matsumoto, ${ }^{b}$ Tatsuhiko Kodama, ${ }^{a}$ and Hideaki Fujitani ${ }^{a}$ \\ ${ }^{a}$ Laboratory for Systems Biology and Medicine, Research Center for Advanced Science and Technology, The \\ University of Tokyo; 4-6-1 Komaba, Meguro-ku, Tokyo 153-8904, Japan: and ${ }^{b}$ Bio-IT R\&D Office, Next-Generation \\ Healthcare Innovation Center, Fujitsu Limited; 1-17-25 Shinkamata, Ota-ku, Tokyo 144-8588, Japan.
}

Received August 19, 2014; accepted December 13, 2014

In this study, we propose a supercomputer-assisted drug design approach involving all-atom molecular dynamics (MD)-based binding free energy prediction after the traditional design/selection step. Because this prediction is more accurate than the empirical binding affinity scoring of the traditional approach, the compounds selected by the MD-based prediction should be better drug candidates. In this study, we discuss the applicability of the new approach using two examples. Although the MD-based binding free energy prediction has a huge computational cost, it is feasible with the latest 10 petaflop-scale computer. The supercomputer-assisted drug design approach also involves two important feedback procedures: The first feedback is generated from the MD-based binding free energy prediction step to the drug design step. While the experimental feedback usually provides binding affinities of tens of compounds at one time, the supercomputer allows us to simultaneously obtain the binding free energies of hundreds of compounds. Because the number of calculated binding free energies is sufficiently large, the compounds can be classified into different categories whose properties will aid in the design of the next generation of drug candidates. The second feedback, which occurs from the experiments to the MD simulations, is important to validate the simulation parameters. To demonstrate this, we compare the binding free energies calculated with various force fields to the experimental ones. The results indicate that the prediction will not be very successful, if we use an inaccurate force field. By improving/validating such simulation parameters, the next prediction can be made more accurate.

Key words computational drug design; molecular dynamics; binding free energy; high-performance computing; force field parameter

As predicted by the Moore's law, ${ }^{1)}$ computational power progresses exponentially each year. The K computer (RIKEN, Japan) was the first one to reach the computational speed of 10 petaflops $(\mathrm{PF}) .{ }^{2)}$ Subsequently, the United States and China released 10 PF-scale supercomputers. Thus, such huge computational power is expected to be utilized effectively for progress in a wide variety of science and technology fields. The ultimate purpose of this study is to develop and demonstrate an efficient drug design method with the help of such a stateof-the-art supercomputer.

To overcome the difficulty of drug development, many computational structure-based drug design (SBDD) methods have been proposed in the last two decades. One important advantage of these computational methods is that they are free of experimental difficulty; thus, the computational methods are expected to reduce the experimental effort involved in the SBDD. The in silico SBDD methods can be categorized into two groups: virtual screening ${ }^{3-8)}$ and de novo drug design. ${ }^{9-11)}$ In virtual screening method, drug candidates are selected from libraries of chemical compounds by predicting their binding free energies approximately. In de novo drug design method, drug candidates are generated from scratch. Although there are various types of de novo drug design methods, they require selection of the best candidates from the designed compound group using the approximate binding free energy calculations. In any computational SBDD methods, the selection of drug candidates plays an important role; therefore, many binding affinity scores have been proposed for improving in silico $\mathrm{SBDD}$.

The success of the computational methods discussed above, however, is largely limited. Warren et $a l^{7)}$ examined several popular SBDD methods by comparing their empirical binding affinity scores with the experimental values. They found that the correlations between the calculated scores and the experimental affinities were not strong enough to distinguish the nM-affinity compounds from lower-affinity compounds. This implies that the SBDD program cannot successfully select the high-affinity compounds from a compound library or a set of designed compounds, although it may not directly indicate that the empirical scoring formulae are inaccurate. There are two potential origins of weak correlation between the empirical scores and the experimental affinities: the fact that the empirical scoring formulae adopt rough approximations and the failure of the binding pose prediction. Even if the empirical scoring formula is accurate, a wrong binding pose could possibly lead to inaccurate binding affinity prediction.

One of the simplest methods for directly assessing the empirical scores is to employ an accurate computational prediction method with the same binding poses instead of experiments. In the previous study, ${ }^{12)}$ we employed the alchemical binding free energy evaluation method, Massively Parallel Computation of Absolute binding Free Energy (MPCAFEE), ${ }^{13,14)}$ and showed that the empirical score (the interaction energy between a ligand and its target protein) is not 
(a)

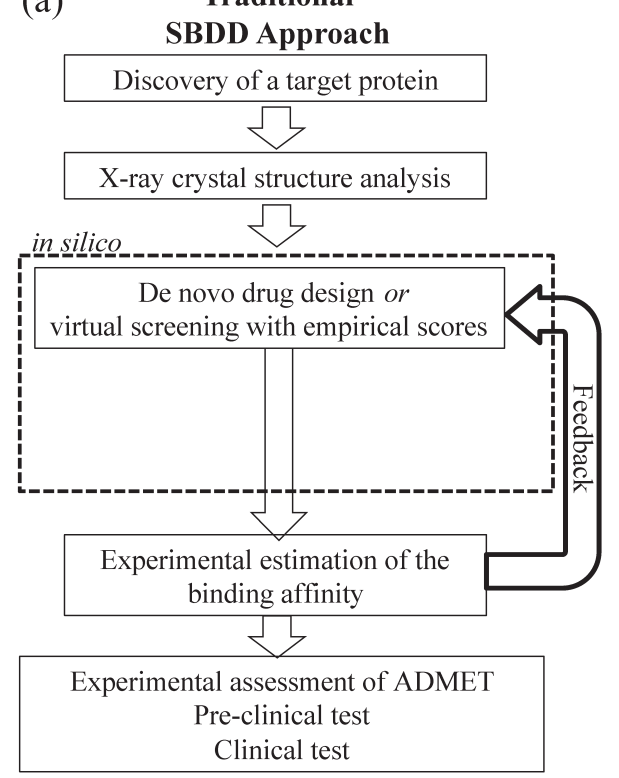

(b)

Supercomputer-assisted

SBDD Approach

Discovery of a target protein
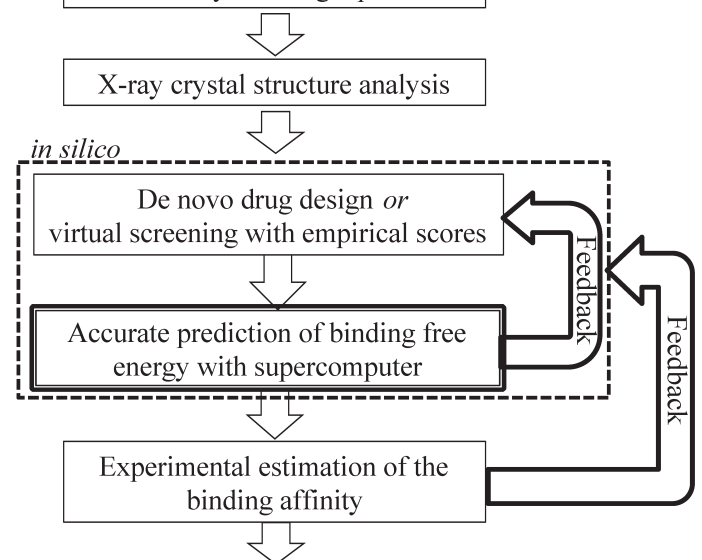

Experimental assessment of ADMET

Pre-clinical test

Clinical test

Fig. 1. Flowcharts of the (a) Traditional SBDD Approach and (b) Supercomputer-Assisted SBDD Approach

strongly correlated with the calculated binding free energy. Because the molecular dynamics (MD) trajectories started from the same binding pose as in the empirical scoring, the calculated free energy and empirical score were compared in the same pose; thus, the result directly indicated the inaccuracy of the empirical scoring (see ref. 12 for more detail). Because the binding free energy is not strongly correlated with any properties calculated from a fixed structure, we consider that dynamical effects are important for the quantitative prediction of binding free energies.

In the MP-CAFEE method, many all-atom MD calculations are performed and free energy differences are calculated using the Jarzynski equality. This method is exact except for the numerical discretization and the employed force field model; therefore, dynamical effects are naturally taken into account. In fact, the MP-CAFEE method predicted absolute values of the binding free energies with an accuracy of $1 \mathrm{kcal} / \mathrm{mol}^{15}{ }^{15}$ In this study, we investigate the possibility that this accurate but computationally heavy prediction method improves the efficiency of computational SBDD.

In the remaining part of this paper, we propose a new SBDD approach involving accurate MP-CAFEE calculations first. To explain possibility of this approach, we introduce two examples: a kinase and pheromone-binding protein. In the first example, we show that the high-performance computer can actually calculate hundreds of binding free energies quickly. This number is large enough to perform the categorization analysis. We consider that the analysis will be an important clue for designing new drug candidates. In the second example, to discuss the validation of the MD-based binding free energy prediction, we calculate binding free energies with various force fields and compare them with the experimental ones. This analysis implies that inappropriate force field will provide inaccurate binding free energy prediction. If we find large deviation between the calculated values and experimental values in the SBDD procedure, it is a good opportunity to improve the simulation parameters including force field parameters. After improving/validating the simulation param- eters, the next prediction will be made more accurate.

\section{Experimental}

Supercomputer-Assisted SBDD Protocol Figure 1 compares our new SBDD protocol with a traditional one. In both cases, the first step is to identify a target protein and measure its three-dimensional coordinates typically with X-ray crystallography. In the traditional method (Fig. 1a), the drug candidates are selected only with the empirical binding scores. Although the calculations of the empirical binding scores can be easily conducted using a standard personal computer, the water molecules are neglected and the protein is assumed to be rigid; these approximations compromise the accuracy of the binding affinity prediction. ${ }^{12)}$ Therefore, the selected compounds rarely show high affinity in the subsequent experiments and many trials are required to discover hit compounds.

In the new method (Fig. 1b), we introduce an accurate MD-based binding free energy prediction step immediately after the conventional in silico drug design step. Because it takes more than one month to experimentally synthesize new compounds from scratch, it should be helpful to predict the binding free energies accurately before the experiments. If the calculated affinities of the designed compounds are lower than expected, we need not conduct the subsequent experiments. Therefore, we expect that this new prediction step to significantly reduce the experimental cost and time. Although this new step requires huge computational resources, a 10 PF-scale supercomputer has sufficient power to perform this protocol, as shown below.

The supercomputer-assisted SBDD approach also involves two important feedback procedures. The first feedback is generated from the MD-based binding free energy predictions to the traditional drug design step. While the experimental feedback in the traditional SBDD approach suggests only tens of designed compounds at most, the MD-based binding free energy calculations in the supercomputer-assisted SBDD approach can be performed for hundreds of compounds at a time. This large number of compounds enables us to cat- 


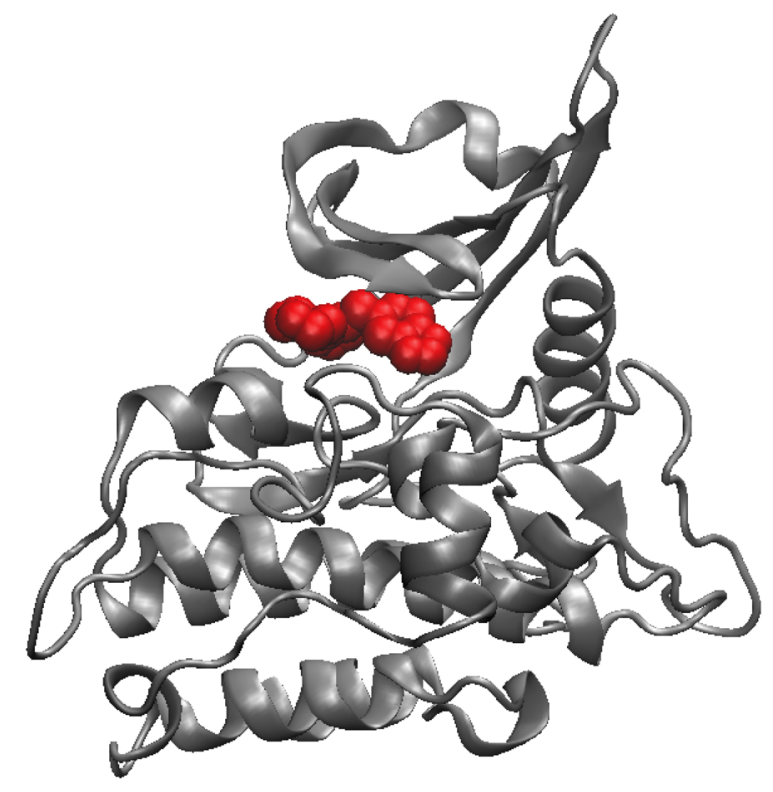

Fig. 2. Structure of the Target Protein; Balls Represent a Compound Designed by OPMF

egorize the compounds and analyze the categories, providing valuable information for the design of the next generation of drug candidates. The second feedback is generated from the experiments to the MD simulations. Because the accuracy of the MD simulation is sensitive to its simulation parameters, it is important to verify whether or not the employed simulation parameters are valid for the simulation systems by comparison with experiments.

Materials and Methods In the new approach, the MDbased binding free energy prediction serves as an accurate fine filter. In this study, we employ MP-CAFEE as the binding free energy prediction method. As discussed in the introduction, previous studies ${ }^{13-15)}$ have already shown the accuracy of the MP-CAFEE method; therefore, this method will help us to efficiently select high-affinity drug candidates. To demonstrate the feasibility of the binding free energy prediction procedure, we adopted the same target protein as that in ref. 12 (Fig. 2). The protein is a typical serine/threonine kinase and a potential therapeutic target of human cancer. The fragmentbased de novo design method, Optimum Packing of Molecular Fragments (OPMF), was used as the traditional drug design method. ${ }^{12)}$ After characterizing the target cavity of the protein, we generated 23 abstract structures. From these abstract structures, 3759 compounds were designed. Subsequently, the polar surface area (PSA), ${ }^{16)}$ lipophilicity (partition function, $\log P),{ }^{17)}$ and synthesizability were examined and 890 of the compounds were selected for the target protein in this study. Because the empirical score was not sufficiently accurate, 299 compounds were randomly selected. Although the number of compounds was decreased, it is still large enough to discuss several important features of the supercomputer-assisted SBDD approach.

Subsequently, we performed three MD simulations to analyze the structural stability of each selected compound. The trajectories were calculated for over $50 \mathrm{~ns}$ under the isothermal-isobaric conditions using the Nose-Hoover thermostat $\left(T=298 \mathrm{~K}, \tau_{\mathrm{T}}=0.5 \mathrm{ps}\right)$ and Berendsen barostat ( $p=1 \mathrm{~atm}$, $\left.\tau_{p}=1 \mathrm{ps}\right)$. Here, the FUJI force field was used for the protein

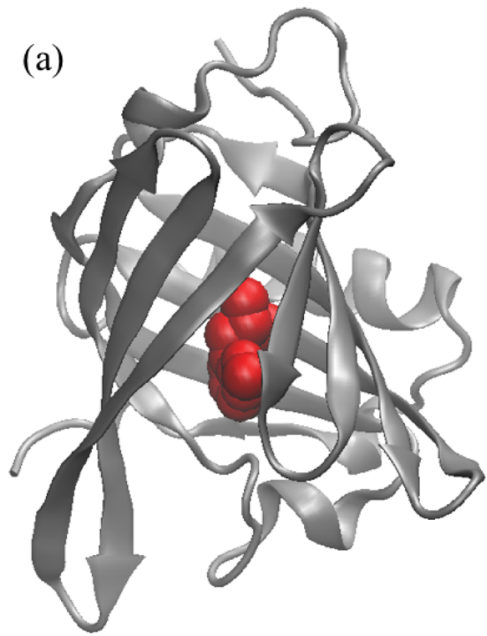

(b)<smiles>COc1nccnc1CC(C)C</smiles>

IBMP

(c)<smiles>COc1nccnc1C(C)C</smiles>

Fig. 3. Structures of (a) MUP-I (Balls Are IBMP Binding in the Hydrophobic Cavity), (b) IBMP, and (c) IPMP

and compounds, and the ligand-protein complex was surrounded by TIP3P water molecules. (See ref. 18 for the details of FUJI.) If the RMSD values between the initial and final compound structures were less than $2.7 \AA$ for all three MD trajectories, we considered the compound to be stably bound to the target protein. An unstable binding pose essentially indicates a weak binding affinity, making further calculation of binding free energy with the MP-CAFEE method unnecessary. In this step, we discarded 30 compounds from the drug candidate list.

The binding free energies of the remaining 269 compounds were calculated with the MP-CAFEE method. For each compound, we calculated 768 independent MD trajectories. Although each trajectory was only simulated for $3 \mathrm{~ns}$, the total simulation time for each compound was longer than $2 \mu$ s and the total simulation time for all candidates exceeded $600 \mu \mathrm{s}$. The initial structures of the MP-CAFEE calculations were taken from the final structures of the preceding MD simulations. The other parameters for the MP-CAFEE calculations were the same as those in ref. 12 .

The new SBDD approach involves the feedback from experiments to the in silico part, which is important to verify the parameters of MD calculations included in the binding free energy evaluation. Accurate simulation parameters are critical to the success of the binding free energy prediction; thus, the simulation parameters must be improved if the experimental values of the binding free energy do not agree with the calculated ones. Although the experimental values of the binding free energy are necessary to demonstrate the importance of this feedback, the experimental data are not available for the kinase studied here unfortunately; therefore, we introduced major urinary protein I (MUP-I) ${ }^{19)}$ (Fig. 3a). This protein has a hydrophobic cavity in which several small molecules can bind. For example, the binding free energy of 2-isobuthyl3-methoxypyrazine (IBMP) (Fig. 3b) was determined by isothermal titration calorimetry; interestingly, the binding free energy of IBMP was $1.1 \mathrm{kcal} / \mathrm{mol}$ larger than that of 2-isopropyl-3-methoxypyrazine (IPMP) (Fig. 3c). Because these two molecules have clearly different binding free energies despite their structural similarity, these systems offer an ideal system for evaluating the binding free energy calculation protocol. 
(a)

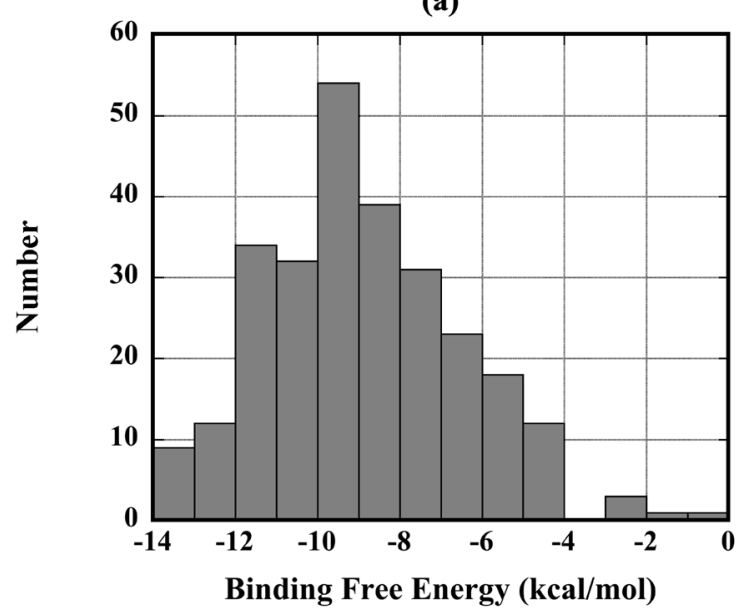

(c)

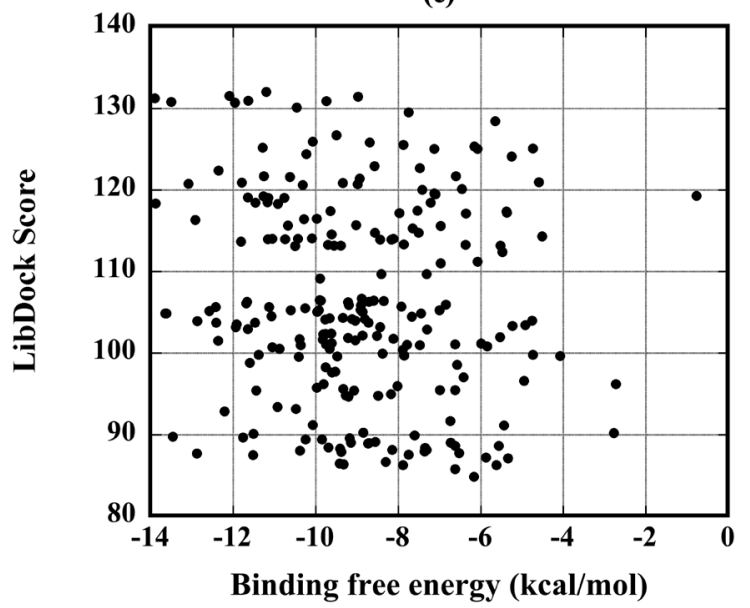

(b)

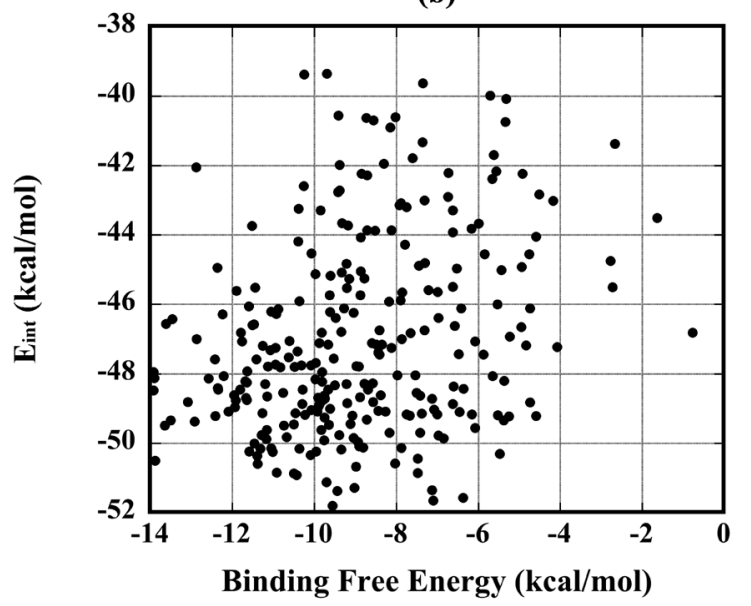

(d)

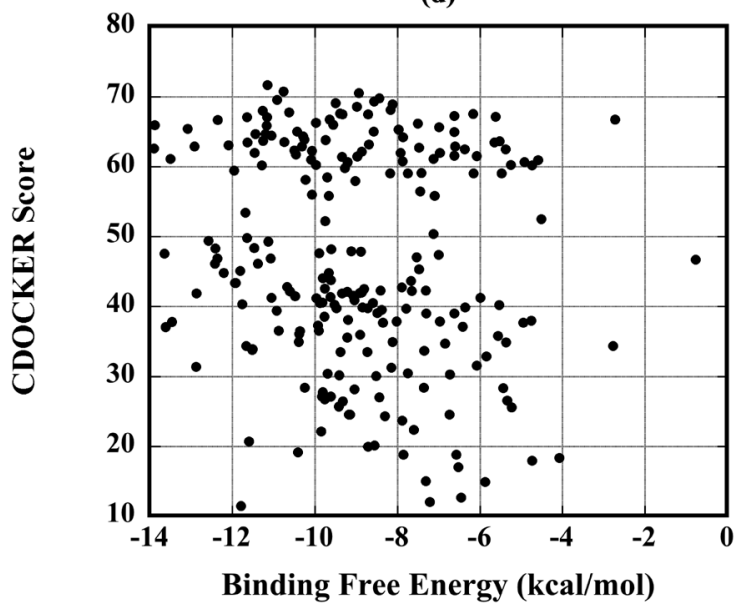

Fig. 4. (a) Distribution of the Binding Free Energies Calculated with MP-CAFEE; (b) Correlation between the Interaction Energies and Binding Free Energies; (c) Correlation between the LibDock Scores and Binding Free Energies; (d) Correlation between the CDOCKER Scores and Binding Free Energies

In this study, we focused on the dependence of the binding free energy prediction on the choice of the protein force field. After $20 \mathrm{~ns}$ MD equilibration with the FUJI force field at $298 \mathrm{~K}$, we construct new systems in which the FUJI force field was replaced with AMBER ff94, ff99, ff99SB, or ff03. These systems were further equilibrated at $308 \mathrm{~K}$ for $50 \mathrm{~ns}$ and the final structures were used as the initial structures in the MPCAFEE calculations. Each trajectory ran for $9 \mathrm{~ns}$ in the constant $\mathrm{N} p \mathrm{~T}$ ensemble. The system temperature was controlled with the Nose-Hoover method $(T=308 \mathrm{~K})$, while the pressure was restrained to 1 atm with the Parrinello-Rahman method. The initial structures of the IBMP and IPMP complexes were taken from the Protein Data Bank (PDB entries 1qyl and 1qy2, respectively). ${ }^{19)}$

All the MD simulations in this study were performed with GROMACS (ver. 4.5.5) ${ }^{20)}$; we ported the program to the new $\mathrm{K}$ computer system particularly for the MP-CAFEE calculations immediately after $\mathrm{K}$ was released.

\section{Results and Discussion}

Massively Parallel Computation of Binding Free Energies To demonstrate the feasibility of the supercomputerassisted SBDD approach (Fig. 1b), we introduced a typical kinase, designed 269 inhibitors for the kinase, and calculated the binding free energies with the MP-CAFEE method. The calculated binding free energies were widely distributed from -14 to $0 \mathrm{kcal} / \mathrm{mol}$ (Fig. $4 \mathrm{a}$ ). Using these values, we can select several best compounds with the chemical accuracy.

The MD-based binding free energy prediction is much more accurate than the empirical scoring or a simple approximation method. The accuracy of the selection method is critical for the success of computational SBDD. If we employ the rigid protein approximation to reduce the computational cost, however, the binding free energy prediction becomes inaccurate. For example, Fig. 4b shows that the interaction energy is not strongly correlated with binding free energy $(R=0.20)$. This result is essentially the same as in ref. 12 . We, therefore, consider that the dynamical effects are necessary to quantitatively predict the binding free energy and that MD-based binding free energy prediction methods will play a key role in making the drug discovery procedure faster and more efficient.

Furthermore, we compared the calculated binding free energies with two popular empirical scores: LibDock ${ }^{21,22)}$ and CDOCKER. $^{23)}$ As shown in Figs. $4 \mathrm{c}$ and $\mathrm{d}$, the correlations between the empirical scores and the binding free energy $(R=-0.11$ and -0.13 for LibDock and CDOCKER, respec- 
(a)

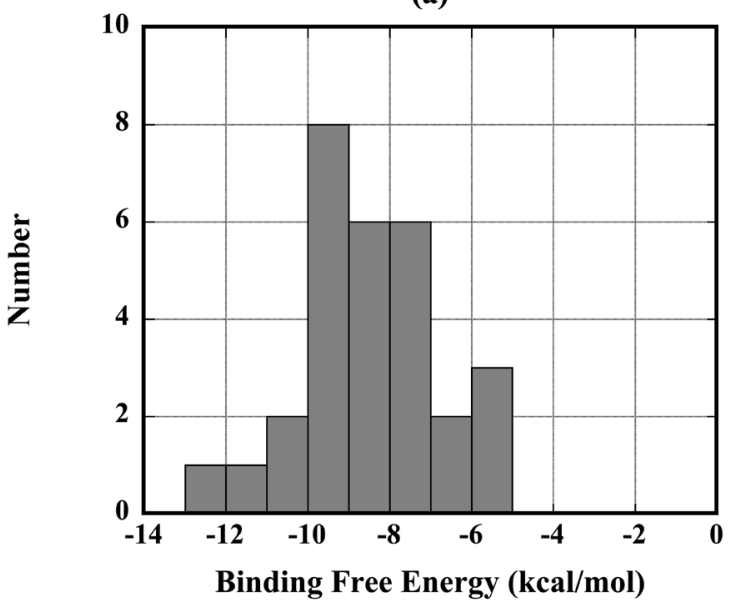

(c)

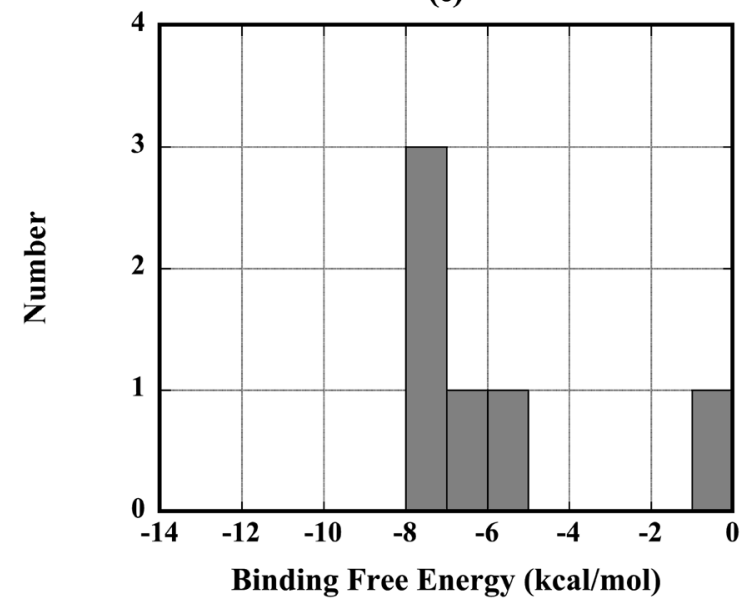

(b)

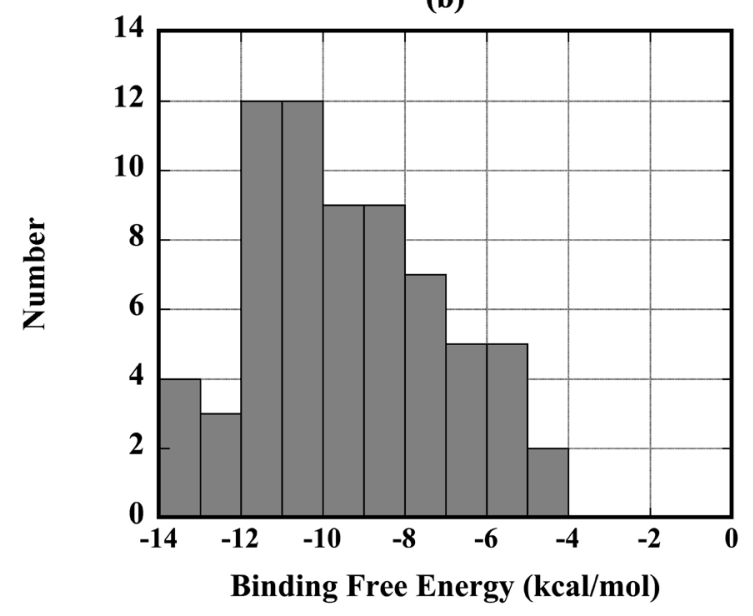

(d)

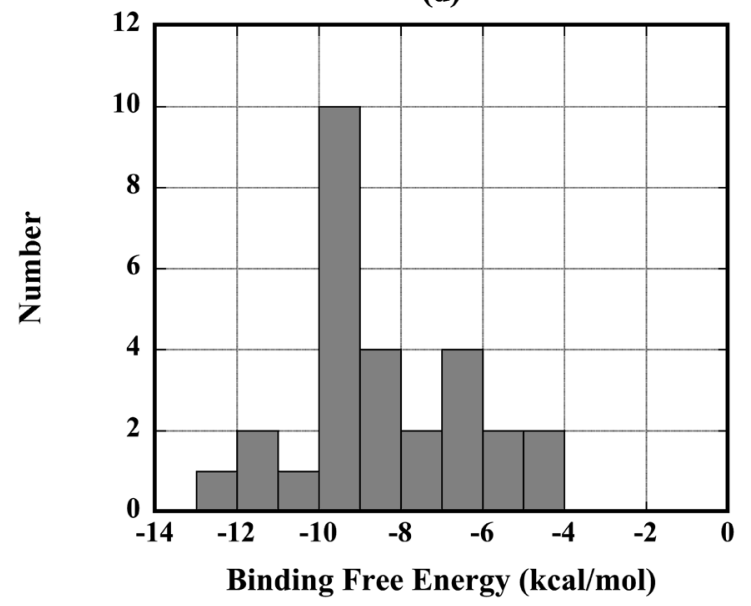

Fig. 5. Distributions of the Binding Free Energies for (a) Skeleton 1, (b) Skeleton 2, (c) Skeleton 3, and (d) Skeleton 4

tively) are as weak as that between the interaction energy and the binding free energy, while these empirical scores are more strongly correlated with the interaction energy. (See Fig. S1. $R=-0.68$ and -0.44 for LibDock and CDOCKER, respectively.) These results indicate that the traditional empirical scores ignore several essential parts of the binding free energy (e.g. binding entropy).

These calculations required a total of $c a .620 \mu \mathrm{s}$ of MD sampling, which is too computationally expensive to conduct on a standard computer. However, a state-of-the-art 10 PFscale computer $(\mathrm{K})$ has become available and we performed the calculations within a few months using $\mathrm{K}$. In the ideal situation where all the nodes of $\mathrm{K}$ are utilized, the calculations would have taken approximately two weeks. This time requirement is not too long relative to the experimental time requirement; it typically takes longer than one month to synthesize new compounds from scratch. Furthermore, at the time of writing this paper, a new version of GROMACS, which includes subroutines optimized for Scalable Processor Architecture (SPARC) chips of the $\mathrm{K}$ computer, was released in collaboration with our team. Thus, new versions of GROMACS (ver. 4.6.1 and later) are two times faster than the present version (ver. 4.5.5) on the $\mathrm{K}$ computer. In the near future, the $10 \mathrm{PF}$-scale computing will become the standard according to Moore's law. Considering these advancements both in hardware and software, we conclude that the proposed SBDD approach is no longer infeasible in practice.

While one advantage of the computational SBDD methods is that they are free of experimental difficulties, improving the accuracy of the binding free energy prediction is also very important. Jorgensen and co-workers developed a Monte Carlo (MC) simulation-based free energy perturbation (FEP) method to predict the relative binding free energies of ligand-protein complexes, ${ }^{24,25)}$ and then proposed a FEP-guided lead optimization. ${ }^{9,26)}$ Although their binding free energy prediction method was not as accurate as MP-CAFEE, it was successfully applied to an actual pharmaceutical target, human immunodeficiency virus (HIV)-1 reverse transferase., ${ }^{97}$ Their results along with those of this study suggest that the all-atom MD or MC simulation-based free energy calculation methods will become a new paradigm in computational drug design.

Another advantage of the accurate in silico prediction of binding free energy is the feedback to the drug design or virtual screening step (Fig. 1b). Although drug design or virtual screening can receive feedback from experiments in the traditional SBDD approach (Fig. 1a), the number of candidates examined experimentally is usually less than 10 , and the feedback is therefore quite limited. In contrast, the supercomputer can calculate binding free energies for more than 100 candidates; in fact, we performed MP-CAFEE calculations for 269 
(a)

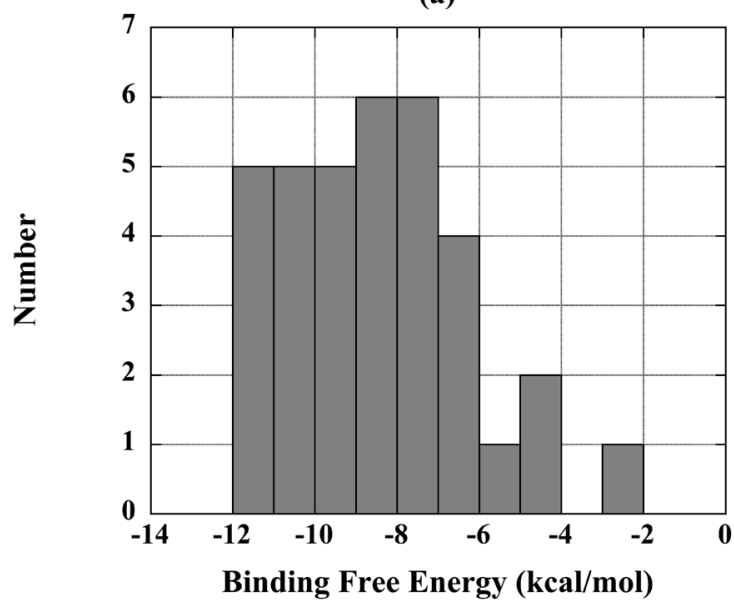

(c)

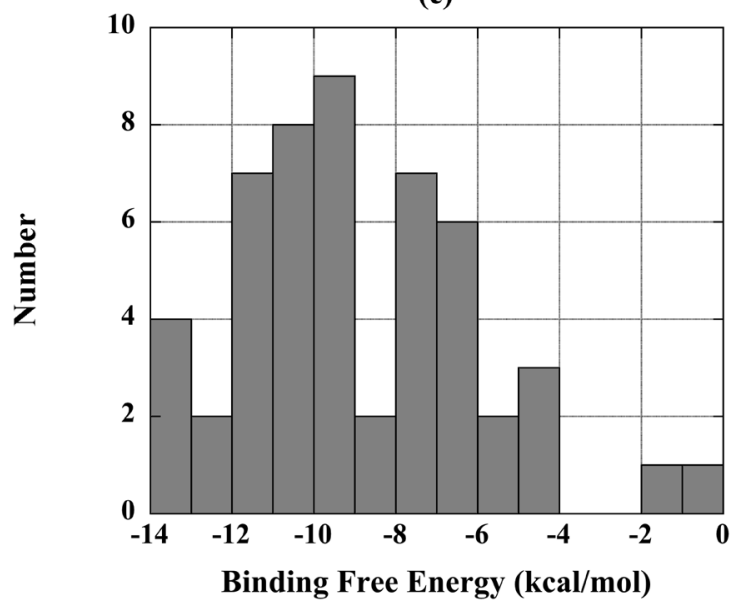

(b)

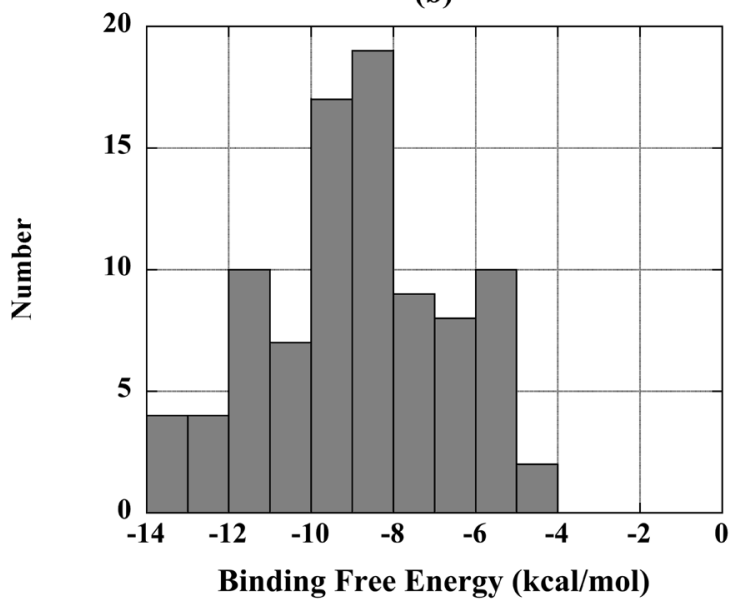

(d)

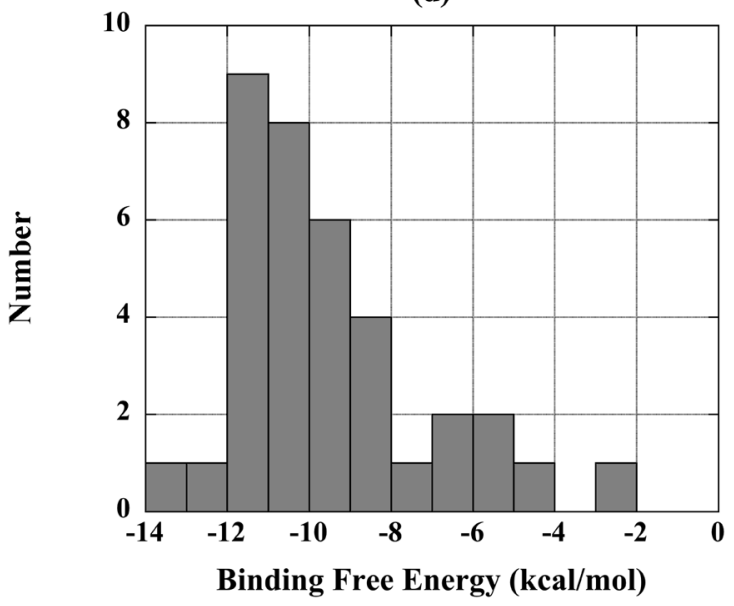

Fig. 6. Distributions of the Binding Free Energies for Designed Compounds Containing Different Numbers of Hydrogen Bonds; (a) Three, (b) Four, (c) Five, and (d) Six

compounds. Because this number is sufficiently large to categorize the compounds into several groups, we can gain insight into the characteristics of the categories. (Although the binding free energies for 58 compounds were calculated in ref. 12 the number is not large enough to characterize each category.)

In the OPMF step, we generated abstract skeletons and modified them to design real compounds. ${ }^{12)}$ Accordingly, the compounds can be naturally categorized depending on the skeleton types. Figure 5 shows the distributions of the calculated binding free energies of the selected skeleton groups. The characteristic shape of each distribution may allow us to decide which skeleton should be given high priority in the next drug design. For example, there are no high-affinity compounds in Fig. 5c, while several compounds have nM affinity in Fig. 5b. In addition, the study on the origin of the wide distribution of each skeleton group will provide deeper insight into the drug design strategy.

Effective categorization can also be done depending on the hydrogen bond number; hydrogen bonding plays an important role in the interaction between the target protein and the compounds. Herein, we classified the compounds into categories depending on the number of the hydrogen bonds counted during the OPMF procedure. Figure 6 shows the distributions of the calculated binding free energies for the given numbers of hydrogen bonds. The average binding free energies were $-8.5,-8.8,-9.0$, and $-9.5 \mathrm{kcal} / \mathrm{mol}$ for the groups with three, four, five, and six hydrogen bonds, respectively, and the ratios of the high affinity compounds with binding free energies exceeding $-11 \mathrm{kcal} / \mathrm{mol}$ were $0.14,0.20,0.25$, and 0.33 , respectively. In this sense, we may conclude that the formation of hydrogen bonds enhances the binding affinity. However, it is important to note that the formation of a new hydrogen bond does not always enhance the binding free energy. For example, hydrogen bonds were determined geometrically in the OPMF process; thus, they were not always maintained in the equilibrated structures. Although more detailed analyses are required to obtain quantitative insight into the drug design, we can conclude here that the above categorizations provide important information for improving the drug design strategy in the next trial.

Validation of the Simulation Parameters As shown in Fig. 1b, the experiments provide feedback to the in silico procedures, possibly providing clues for the design of new drug candidates in the same manners as in the traditional SBDD approach (Fig. 1a). Furthermore, we can check the simulation parameters by comparing the experimental results with the computational prediction. In this section, we focus on the influence of the force field parameters. We calculated the bind- 
Table 1. Binding Free Energy of MUP-I (kcal/mol)

\begin{tabular}{lccc}
\hline \hline & $\Delta G_{\text {bind }}($ IBMP $)$ & $\Delta G_{\text {bind }}($ IPMP $)$ & $\Delta \Delta G_{\text {bind }}$ \\
\hline FUJI & $9.0 \pm 0.1$ & $8.2 \pm 0.1$ & $+0.8 \pm 0.1$ \\
ff94 & $7.2 \pm 0.1$ & $6.4 \pm 0.1$ & $+0.8 \pm 0.1$ \\
ff99 & $9.5 \pm 0.1$ & $9.8 \pm 0.1$ & $-0.3 \pm 0.1$ \\
ff99SB & $9.2 \pm 0.1$ & $8.6 \pm 0.1$ & $+0.6 \pm 0.1$ \\
ff03 & $8.5 \pm 0.1$ & $7.7 \pm 0.1$ & $+0.8 \pm 0.2$ \\
Exptl. $^{a)}$ & 9.2 & 8.1 & +1.1 \\
\hline
\end{tabular}

a) Experimental data are taken from ref. 19 (Bingham et al., JACS, 2004).

ing free energies of the MUP-I systems and compared them with the experimental ones.

The calculated binding free energies for various force fields are tabulated along with the experimental values in Table 1. For the FUJI force field, the absolute binding free energies agreed with the experimental ones. While the relative binding free energy $\left(\Delta \Delta G_{\text {bind }}\right)$ is $+0.8 \mathrm{kcal} / \mathrm{mol}$ for the FUJI force field, the experimental value is $+1.1 \mathrm{kcal} / \mathrm{mol}$. The ff99SB results slightly deviated from the experimental data slightly, although they are acceptable for the present purpose. The ff99 force field significantly underestimated the relative binding free energy $\left(\Delta \Delta G_{\text {bind }}=-0.3 \mathrm{kcal} / \mathrm{mol}\right)$ due to the large overestimation of the absolute binding free energy of IPMP. In the other cases (ff94 and ff03), the relative binding free energies were similar to the experimental value, however the absolute binding free energies were underestimated.

If we start the supercomputer-assisted SBDD procedure with ff99 for this system, the binding free energy prediction is less accurate and the drug candidate selection is less successful. However, the experimental feedback of the supercomputer-assisted SBDD procedure (Fig. 1b) will call our attention to the inaccuracy. Then, we can improve the force field parameters or replace ff99 with a better force field and the binding free energy prediction of the next selection step can be made more accurate.

The deviation in the calculated binding free energies from the experimental ones is largely attributed to the inaccurate modeling of the force fields. Despite of the advances in wide variety of computational methods, the modeling of the force field is not so easy and there is room for improvement of force field. For example, the force field parameters for proteins are not perfectly consistent with those for small compounds in the standard AMBER-style simulations ${ }^{14)}$; the small compounds are described by the general AMBER force field (GAFF), which employs the different atom type classifications (Tables S1 and S2). In addition, it was difficult to clearly determine the torsion force field parameters for $\phi$ and $\psi$ angles. The potential energy profiles along the $\phi$ and $\psi$ angles are strongly dependent on the choice of the force field. ${ }^{15,18)}$ In contrast to the bond and angle vibrational motions, most torsional motions are unlike harmonic vibrations and can cause conformational changes. Although the experimental values of the vibrational frequencies are accurate, it is difficult for experiments to determine the activation energy for conformational changes. Furthermore, owing to the intramolecular throughspace interaction, the standard-level $a b$ initio calculations cannot calculate an energy profile along the $\phi$ and $\psi$ angles accurately $^{18)}$ (Fig. S2).

The development of the FUJI force field was essentially the same as the experimental feedback procedure of the
Table 2. Conformational Distribution of Ala Dipeptide

\begin{tabular}{lcccc}
\hline \hline & $\alpha_{\mathrm{R}}$ & $\beta$ & $\mathrm{P}_{\mathrm{II}}$ & Others \\
\hline $\mathrm{FUJI}^{a)}$ & $0.059 \pm 0.002$ & $0.226 \pm 0.002$ & $0.706 \pm 0.005$ & $0.008 \pm 0.004$ \\
$\mathrm{ff9}^{a}$ & $0.867 \pm 0.013$ & $0.030 \pm 0.005$ & $0.095 \pm 0.010$ & $0.007 \pm 0.003$ \\
$\mathrm{ff99}^{a)}$ & $0.880 \pm 0.006$ & $0.062 \pm 0.004$ & $0.019 \pm 0.000$ & $0.038 \pm 0.003$ \\
$\mathrm{ff99SB}^{a)}$ & $0.264 \pm 0.005$ & $0.277 \pm 0.006$ & $0.440 \pm 0.003$ & $0.018 \pm 0.010$ \\
$\mathrm{ff03}^{a}$ & $0.420 \pm 0.020$ & $0.196 \pm 0.010$ & $0.373 \pm 0.012$ & $0.011 \pm 0.001$ \\
$\mathrm{IR}^{b)}$ & 0.11 & 0.29 & 0.60 & - \\
Raman $^{b)}$ & 0.09 & 0.29 & 0.62 & -
\end{tabular}

a) The values were calculated from three $100 \mathrm{~ns}$ MD simulations at $298 \mathrm{~K}$ and $1 \mathrm{~atm}$. b) The experimental data are taken from ref. 30 (Grdadolnik et al., PNAS, 2011).

supercomputer-assisted SBDD. (See ref. 18 for the details of FUJI.) In fact, Fujitani and his coworkers carefully improved the force field parameters from the viewpoint discussed above. This may be a reason the binding free energies calculated by the MP-CAFEE method along with FUJI is in the best agreement with the experimental ones.

In addition, a disinterested group has already shown that FUJI can reproduce the experimental results of conformational distributions better than the other standard force fields (CHARMM27, CHARMM36, ff99SB, OPLS-AA/L, and GROMOS 54a7), although the CHARMM36 and OPLS-AA/L are improved considerably. ${ }^{28)}$ We also showed that the conformational distribution in the aqueous phase is sensitive to the choice of the force field, by performing MD simulations of the Ala dipeptide. The calculated distributions are consistent with previous results. ${ }^{15,29)}$ In this study, the fractional populations were calculated using the same conformational basins definitions as those in ref. 29. As in Table 2, while the results of the FUJI force field were in good agreement with the experimental results, the other force fields (ff94, ff99, ff99SB, and ff03) largely overestimated the population of the $\alpha_{\mathrm{R}}$ basin and underestimated those of the $\beta$ and $\mathrm{P}_{\mathrm{II}}$ basins. ${ }^{30)}$ More interestingly, Beauchamp et al. ${ }^{31)}$ examined 11 recent force fields (ff96, ff99, ff03, ff03*, ff03w, ff99SB*, ff99SB-ILDN, ff99SB-ILDN-PHI, ff99SB-ILDN-NMR, CHARMM27, and OPLS-AA) for accuracy and showed that all of them resulted in the overestimation of $\alpha_{\mathrm{R}}$ and underestimation of the $\beta$ and $\mathrm{P}_{\mathrm{II}}$ in dipeptides (Fig. S25 of ref. 31). These results support the superiority of FUJI over the other force fields for now, although we may develop a better force field in future. The inaccuracy of the force field indicates that the long MD simulation of protein will result in wrong equilibrated structures. This may enhance the error in the binding free energy prediction, because the MP-CAFEE method requires a correctly equilibrated structure.

In addition to the choice of the force fields, the MD-based prediction method is also affected by the running time, the initial pose, the number of sampling runs, and so on. ${ }^{12,15,32)}$ In particular, the protein structure is very fragile, hence we need to carefully prepare the initial simulation conditions. ${ }^{33}$ ) Furthermore, we may sometimes need to check the quality of the X-ray crystal structure. ${ }^{34)}$ As we have demonstrated for several spectroscopic results, ${ }^{35-37)}$ many experiments face difficulties for interpreting their results. Because computer simulation can help to correctly interpret the experiments, MD simulation will be essential in making the drug design procedure more logical and efficient. In summary, the latter 
demonstration explains the experimental feedback; the calculated results should be compared with the experimental ones a few times in the supercomputer-assisted SBDD procedure (Fig. 1b). Any observed deviations essentially indicate that the simulation parameters need to be modified to improve the accuracy of the binding free energy prediction.

\section{Conclusion}

In this study, we introduced a novel supercomputer-assisted SBDD approach, in which a $10 \mathrm{PF}$-scale high performance computer is utilized for the acceleration of the MD-based binding free energy predictions. In the traditional SBDD approach, the binding free energy is estimated using two rough approximations: the target protein is rigid and the water molecules are not considered explicitly. Although these simplifications allow the calculation of the binding free energies of many compounds without great computational cost, the predictions are inaccurate. In contrast, the supercomputerassisted SBDD approach employs MD-based binding free energy predictions, in which all atoms including those of water are modeled at the atomic level. The MD simulations can thus reproduce the natural motion, and conformational sampling and entropic effects are accurately taken into account. The downside of this method is that calculations require a huge computational cost. Thus, we used the $\mathrm{K}$ computer to perform MP-CAFEE calculations for hundreds of designed compounds. In the present demonstration, a few months were required to complete all the calculations using only a small part of computational resources of $\mathrm{K}$. This time frame is not too long relative to the time scale of the actual drug design process. Because the accuracy of the prediction strengthens the filtering function, we expect that the supercomputer-assisted SBDD approach can reduce the experimental load (i.e., expensive cost and manpower).

Furthermore, we discussed that the supercomputer-assisted SBDD approach involves two important procedures that make the drug design process more efficient and effective. The first one is the feedback generated from the accurate binding free energy prediction to the drug design strategy. Because the high-performance computer provides information about hundreds of designed compounds at once, the compounds can be classified into several categories, and the characteristics of each category can be identified. Although the feedback in the traditional SBDD approach is generated from the experiments to the drug design, the number of compounds that can be measured at one time is limited and thus it is difficult to characterize the categorization of the compounds accurately. In fact, we showed that the state-of-the-art computer not only strengthened the filtering function but also provided several new clues for improving the next drug design strategy in this study. The second one is the feedback generated from the experiments to the in silico procedures. In this study, we showed that the choice of the force field influenced the binding free energy prediction significantly. If we start the supercomputer-assisted SBDD procedure with an inaccurate force field, we will find at the step of experimental check that some calculated binding free energies deviate significantly from the experimental ones. Before the next drug design step, we can improve the accuracy of the prediction by replacing the force field with a better one or modifying the parameters; thus, the next selection step of drug candidates will be made more successful. In addition, other simulation parameters such as equilibration time, initial pose, run time, and number of sampling runs can also affect the binding free energy prediction. For this reason, collaboration with experimentalists is critical in this field, and we are thus working on additional demonstrations of the supercomputer-assisted SBDD approach in collaboration with several experimental teams.

Acknowledgments This research was partly funded by MEXT SPIRE Supercomputational Life Science (hp120297, hp130006, and hp140228) and partly by the FIRST Kodama project. In particular, the huge computational resources of the $\mathrm{K}$ computer (RIKEN, Japan) enabled us to perform all the MP-CAFEE calculations.

Conflict of Interest The authors declare no conflict of interest.

Supplementary Materials The online version of this article contains supplementary materials.

\begin{tabular}{|c|c|}
\hline & \\
\hline 1) & Moore G. E., Proc. IEEE, 86, 82-85 (1998). \\
\hline 2) & TOP500. “TOP500 Super computer Sites.”: 〈http://www.top500.org〉. \\
\hline 3) & Blaney J., J. Comput. Aided Mol. Des., 26, 13-14 (2012). \\
\hline 4) & Smith R. D., Dunbar J. B. Jr., Ung P. M.-U., Esposito E. X., Yang \\
\hline & C.-Y., Wang S., Carlson H. A., J. Chem. Inf. Model., 51, 2115-2131 \\
\hline & (2011). \\
\hline 5) & Enyedy I. J., Egan W. J., J. Comput. Aided Mol. Des., 22, 161-168 \\
\hline & $(2008)$ \\
\hline 6) & McInnes C., Curr. Opin. Chem. Biol., 11, 494-502 (2007). \\
\hline 7) & Warren G. L., Andrews C. W., Capelli A.-M., Clarke B., LaLonde \\
\hline & J., Lambert M. H., Lindvall M., Nevins N., Semus S. F., Senger S., \\
\hline & Tedesco G., Wall I. D., Woolven J. M., Peishoff C. E., Head M. S., \\
\hline & J. Med. Chem., 49, 5912-5931 (2006). \\
\hline 8) & Shoichet B. K., Nature (London), 432, 862-865 (2004). \\
\hline 9) & Jorgensen W. L., Acc. Chem. Res., 42, 724-733 (2009). \\
\hline 10) & Schneider G., Hartenfeller M., Reutlinger M., Tanrikulu Y., Pros- \\
\hline & chak E., Schneider P., Trends Biotechnol., 27, 18-26 (2009). \\
\hline 11) & Schneider G., Fechner U., Nat. Rev. Drug Discov., 4, 649-663 \\
\hline & $(2005)$ \\
\hline 12) & Yamashita T., Ueda A., Mitsui T., Tomonaga A., Matsumoto S., Ko- \\
\hline & dama T., Fujitani H., Chem. Pharm. Bull., 62, 661-667 (2014). \\
\hline 13) & Fujitani H., Tanida Y., Ito M., Jayachandran G., Snow C. D., Shirts \\
\hline & M. R., Sorin E. J., Pande V. S., J. Chem. Phys., 123, 084108 (2005). \\
\hline 14) & Fujitani H., Tanida Y., Matsuura A., Phys. Rev. E Stat. Nonlin. Soft \\
\hline & Matter Phys., 79, 021914 (2009). \\
\hline 15) & Fujitani H., Shinoda K., Yamashita T., Kodama T., J. Phys. Conf. \\
\hline & Ser., 454, 012018 (2013). \\
\hline 16) & Ertl P., Rohde B., Selzer P., J. Med. Chem., 43, 3714-3717 (2000). \\
\hline & Wildman S. A., Crippen G. M., J. Chem. Inf. Comput. Sci., 39, \\
\hline & $868-873(1999)$ \\
\hline 18) & Fujitani H., Matsuura A., Sakai S., Sato H., Tanida Y., J. Chem. \\
\hline & Theory Comput., 5, 1155-1165 (2009). \\
\hline 19) & Bingham R. J., Findlay J. B. C., Hsieh S.-Y., Kalverda A. P., Kjell- \\
\hline & berg A., Perazzolo C., Phillips S. E. V., Seshadri K., Trinh C. H., \\
\hline & Turnbull W. B., Bodenhausen G., Homans S. W., J. Am. Chem. Soc., \\
\hline & 126, $1675-1681(2004)$ \\
\hline 20) & Hess B., Kutzner C., van der Spoel D., Lindahl E., J. Chem. Theory \\
\hline & Comput., 4, 435-447 (2008). \\
\hline & Diller D. J., Merz K. M. Jr., Proteins, 43, 113-124 (2001). \\
\hline & Rao S. N., Head M. S., Kulkarni A., LaLonde J. M., J. Chem. Inf. \\
\hline & Model., 47, 2159-2171 (2007). \\
\hline & Wu G., Robertson D. H., Brooks C. L. 3rd, Vieth M., J. Comput. \\
\hline
\end{tabular}


Chem., 24, 1549-1562 (2003).

24) Essex J. W., Severance D. L., Tirado-Rives J., Jorgensen W. L., J. Phys. Chem. B, 101, 9663-9669 (1997).

25) Pierce A. C., Jorgensen W. L., Angew. Chem. Int. Ed. Engl., 36, 1466-1469 (1997).

26) Kim J. T., Hamilton A. D., Bailey C. M., Domoal R. A., Wang L., Anderson K. S., Jorgensen W. L., J. Am. Chem. Soc., 128, 1537215373 (2006)

27) Jorgensen W. L., Ruiz-Caro J., Tirado-Rives J., Basavapathruni A., Anderson K. S., Hamilton A. D., Bioorg. Med. Chem. Lett., 16, $663-667$ (2006).

28) Tzanov A. T., Cuendet M. A., Tuckerman M. E., J. Phys. Chem. B, 118, 6539-6552 (2014).

29) de M. Seabra G., Walker R. C., Roitberg A. E., J. Phys. Chem. A,
113, 11938-11948 (2009).

30) Grdadolnik J., Mohacek-Grosev V., Baldwin R. L., Avbelj F., Proc. Natl. Acad. Sci. U.S.A., 108, 1794-1798 (2011).

31) Beauchamp K. A., Lin Y.-S., Das R., Pande V. S., J. Chem. Theory Comput., 8, 1409-1414 (2012).

32) Okada O., Yamashita H., Takedomi K., Ono S., Sunada S., Kubodera H., Biophys. Chem., 180-181, 119-126 (2013).

33) Yamashita T., Fujitani H., Chem. Phys. Lett., 609, 50-53 (2014).

34) Warren G. L., Do T. D., Kelley B. P., Nicholls A., Warren S. D., Drug Discov. Today, 17, 1270-1281 (2012).

35) Yamashita T., Kato S., J. Chem. Phys., 121, 2105-2116 (2004).

36) Yamashita T., Kato S., Chem. Phys. Lett., 405, 142-147 (2005)

37) Yamashita T., Takatsuka K., J. Chem. Phys., 126, 074304 (2007). 